\title{
The effect of instruction to synchronize over step frequency while walking with auditory cues on a treadmill
}

\author{
Catarina Mendonça ${ }^{\mathrm{a}, \mathrm{b}, \mathrm{c}, *}$, Marta Oliveira ${ }^{\mathrm{d}, \mathrm{e}}$, Liliana Fontes ${ }^{\mathrm{f}}$, Jorge Santos ${ }^{\mathrm{c}, \mathrm{d}, \mathrm{g}}$ \\ a Aalto University, Department of Signal Processing and Acoustics, Finland \\ ${ }^{\mathrm{b}}$ Carl von Ossietzky University, Cluster of Excellence Hearing4all, Germany

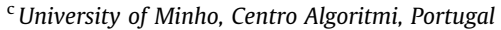 \\ ${ }^{\mathrm{d}}$ University of Minho, Department of Basic Psychology, Portugal \\ ${ }^{\mathrm{e}}$ University of Algarve, Department of Psychology and Educational Sciences, Portugal \\ ${ }^{\mathrm{f}}$ University of Minho, School of Engineering, Centro de Ciência e Tecnologia Têxtil, Portugal \\ ${ }^{\mathrm{g}}$ Centro de Computação Gráfica, Department of Perception, Interaction and Usability, Portugal
}

\section{A R T I C L E I N F O}

\section{Article history:}

Available online 16 January 2014

\section{psycINFO classification:}

2300

2330

3295

3297

Keywords:

Walking

Motor processes

Synchronization

Frequency

Treadmill

Intention

\section{A B S T R A C T}

Walking to a pacing stimulus has proven useful in motor rehabilitation, and it has been suggested that spontaneous synchronization could be preferable to intentional synchronization. But it is still unclear if the paced walking effect can occur spontaneously, or if intentionality plays a role. The aim of this work is to analyze the effect of sound pacing on gait with and without instruction to synchronize, and with different rhythmic auditory cues, while walking on a treadmill.

Firstly, the baseline step frequency while walking on a treadmill was determined for all participants, followed by experimental sessions with both music and footstep sound cues. Participants were split into two groups, with one being instructed to synchronize their gait to the auditory stimuli, and the other being simply told to walk. Individual auditory cues were generated for each participant: for each trial, cues were provided at the participant's baseline walking frequency, at $5 \%$ and $10 \%$ above baseline, and at $5 \%$ and $10 \%$ below baseline.

This study's major finding was the role of intention on synchronization, given that only the instructed group synchronized their gait with the auditory cues. No differences were found between the effects of step or music stimuli on step frequency.

\footnotetext{
* Corresponding author at: Aalto University, Department of Signal Processing and Acoustics, PO Box 13000, FI-00076 Aalto, Finland. Tel.: +358 503677457.

E-mail address: catarina.hiipakka@aalto.fi (C. Mendonça).
} 
In conclusion, without intention or cues that direct the individual's attention, spontaneous gait synchronization does not occur during treadmill walking.

(C) 2013 Elsevier B.V. All rights reserved.

\section{Introduction}

Body motion, including gait, can be influenced by rhythmic sounds. Music, for example, is the external source of information that aerobics practitioners and dance pairs use in order to coordinate their movements (Styns, Van Noorden, Moelants, \& Leman, 2007). This paced movement enabled by external stimuli presents great potential for a wide variety of fields, including education, sports and rehabilitation. The scope of this work lies within the influences of intention on the effect of sound over human gait. Throughout this article, the terms spontaneous- and unintentional synchronization are used interchangeably, referring to a lock to an external stimulus in time and/or frequency without deliberately deciding to do so, or without intention. Conversely, intentional- and forced synchronization refer to gait lock to an external stimulus in time and/or frequency with intention (intentional) or specifically because of explicit instruction/training/constraints (forced). Motor stabilization is used to refer to reduced gait variability either in velocity, frequency, or step amplitude.

In the clinical field, walking with the pacing of rhythmic sounds has been used widely and successfully as a strategy in the motor rehabilitation of some pathological conditions such as stroke (Roerdink, Lamoth, Kwakkel, van Wieringen, \& Beek, 2007; Roerdink et al., 2009), hemiparesis (Pelton, Johannsen, Chen, Chen, \& Wing, 2010), Parkinson's disease (de Bruin et al., 2010; Picelli et al., 2010; Rochester et al., 2007), and Huntington's disease (Thaut, Miltner, Lange, Hurt, \& Hoemberg, 1999). Such rehabilitation approaches can be implemented both in overground and in treadmill walking. Harris-Love, Forrester, Macko, Silver, and Smith (2011) compared the gait patterns of chronic hemiparetic stroke patients during overground and treadmill walking. They found that treadmill induces immediate alterations toward more consistent and symmetric gait patterns, unlike overground walking. As a result, it has been suggested that treadmill might be preferable to overground walking in therapeutic approaches. Combined therapeutic methods, including both treadmill and overground walking, have also revealed positive results in motor rehabilitation (Ada, Dean, Hall, Bampton, \& Crompton, 2003).

Overground walking exhibits a greater potential to be modulated by external cues, since it allows for unconstrained movement. Conversely, walking on a treadmill allows for large periods of motion in highly controllable conditions. However, in treadmill walking there is a limit to the individual's range of step frequency and amplitude due to the treadmill's imposed speed and carpet size. When instructed to synchronize their gait with auditory cues, individuals are forced to change their walking parameters, which may require them to abandon their comfort frequency (Parvataneni, Ploegi, Olney, \& Brouwer, 2009). Nessler and Gilliland (2010) demonstrated that forced synchronization to auditory stimuli while walking on a treadmill resulted in steps that were significantly smaller and faster when compared to independent, unintentional synchronization. The authors concluded that unintentional synchronization might be preferable to intentional synchronization in certain subjects. However, in that study, subjects synchronized to other walkers, which might be unpractical to implement in rehabilitation contexts.

The role of intention in motor synchronization has been studied by presenting individuals with different instructions, either by explicitly asking them to synchronize their gait or by just allowing individuals to walk freely side-by-side (Nessler \& Gilliland, 2009, 2010). Unintentional interpersonal synchronization has been shown to occur often in free walking (Zivotofsky, Gruendlinger, \& Hausdorff, 2012; Zivotofsky \& Hausdorff, 2007). On a treadmill it has been mostly observed in the context of interpersonal walking, but not for all participants (Nessler \& Gilliland, 2010), and although it decreased when visual or auditory interpersonal information was limited (Nessler \& Gilliland, 2009). A study by Sejdić, Jeffery, Kroonberg, and Chan (2012) also found some effect of sounds on treadmill 
walking. In that study, they found that watching television with sounds had an effect on stride stability, leading to greater variability.

In sum, despite some evidence that spontaneous synchronization to auditory stimuli might be useful in rehabilitation contexts, it is not clear whether or not it can occur. Do subjects change their comfort walking pace on a treadmill simply by being presented with rhythmic stimuli? Or are instructions required? Understanding such mechanisms might impact the design of paced walking therapy settings. If spontaneous effects occur, then free exercises might be preferable and, to a certain point, the presence of the therapist can be optional. If intention is required, then some special care has to be taken, namely with patients with attentional deficits, low contextual awareness, or other limitations.

In this work, we defined as primary goal to identify if intention is required to observe an effect of external rhythmic cues while walking on treadmill, either by synchronization or motor stabilization. Because spontaneous interpersonal synchronization has been reported in treadmill walking, a secondary goal was devised to compare rhythmic human steps and music. Therefore, we intended to identify if there would be greater tendency to engage with human step sounds than with abstract rhythms. To better compare the effect of intention on motor adaptation, two experimental groups were tested: one group was clearly instructed to synchronize their gait to the auditory stimuli, while the other group was simply instructed to walk, with no reference being made to the stimuli.

Results are analyzed in terms of participant step frequency in three sections: (1) Effects of velocity, instruction and cue type; (2) Effect of cue frequency value; and (3) Step frequency variations over time.

\section{Methods}

\subsection{Participants}

Nine healthy individuals ( 3 females and 6 males with a mean age of 25 and a mean height of $1.67 \mathrm{~m}$ ) were recruited from the local student population at the University of Minho to participate in this study. None suffered from any musculoskeletal impairment, and all reported to have no known uncorrected visual or hearing impairment.

All participants were volunteers and provided informed consent. Prior to the experiments, the researcher explained that the objective was to study the characteristics of gait, and committed to reporting the results to them at the end of the project. After the experiment ended, a post-investigation clarification revealed to the volunteers that the specific goal was to focus on the effect of stimuli and instructions on step frequency.

\subsection{Materials and settings}

The auditory stimuli of footsteps were generated with Matlab ${ }^{\circledR}$ from a recording of steps on a wooden floor. The music stimuli were produced with the software Cool Edit Pro and mixed on the software BPM Studio 4 Profi.

Prior to the experiment itself, reflective markers were attached to the participants' shoes with Velcro strips on the lateral malleoli of the tibia, on the heels, and on the toes of both feet. All participants were instructed to wear comfortable shoes and were provided with tights that did not interfere with the capture of markers or with normal gait.

The treadmill's palm rest was removed in order to prevent motor interferences on the participants' task. To ensure their safety, individuals were secured with a harness to a lifeline suspended from the ceiling.

During the experiment, the reflective markers were tracked by a 6-camera motion capture system (Vicon MX3+, MXF20) at $120 \mathrm{~Hz}$. Afterwards, the sagittal plane step trajectories were exported and analyzed offline with two algorithms implemented in Matlab ${ }^{\circledR}$.

Finally, a three-computer cluster controlled both the presentation of auditory cues and the speed of the treadmill. 


\subsection{Procedure}

All participants began this study with a preliminary test, where they were instructed to walk on the treadmill without auditory cues. This differed from the main experiment, in which both music and step sounds were presented.

This test consisted of four separate data acquisitions each lasting $120 \mathrm{~s}$, in which participants walked on the treadmill at speeds of $1.0,1.2,1.4$ and $1.6 \mathrm{~m} / \mathrm{s}$. Recording of data started $15 \mathrm{~s}$ after the participant started walking in order to guarantee they had enough time to adjust their gait to the treadmill's speed. Participants were instructed to walk as normally and comfortably as possible, as if they were strolling down the street.

This test provided baseline values on each participant's step frequency and length for each velocity, information that was then used to create the auditory stimuli for the main experiment.

Participants were then placed in one of two groups for the main experiment: the instruction group was told to synchronize their gait with the auditory cues; the no instruction group was given the same instructions as in the preliminary test, and therefore no specific instruction was given to synchronize step frequency with the cues.

Auditory cues consisted of rhythmic sounds of either music or human footsteps, at a constant, and therefore predictable, pace. Stimuli were presented at $74.6 \mathrm{~dB}$ SPL. The sounds could take five different frequency values determined individually for each participant: the baseline step frequency (100\%), step frequencies $5 \%$ and $10 \%$ above baseline (105\% and $110 \%$ ), and step frequencies $5 \%$ and $10 \%$ below baseline (95\% and $90 \%$ ). All of these five cue values were repeated for each of the four treadmill velocities $(1,1.2,1.4$ and $1.6 \mathrm{~m} / \mathrm{s})$ for both music and footstep stimuli, thus totaling 40 different conditions. The five frequency values and type of auditory cue were randomized within each velocity. trial.

Each trial lasted for $135 \mathrm{~s}$, with the auditory cue being presented $15 \mathrm{~s}$ after the beginning of the

\subsection{Statistical analyses}

A Shapiro-Wilk analysis was conducted to assess the normality of the cases to be statistically tested. A mixed Multifactorial ANOVA was performed to assess the effect of interaction between the variables velocity, cue value, cue type and instruction over dependent variable step frequency $(4 \times 5 \times 2 \times 2)$. Additionally, a One Way ANOVA was performed comparing the mean step frequencies per instruction group.

To better analyze the effect of cue frequency value on step frequency, Pearson correlation tests were performed between the two variables with pooled data points from all observations $(d f=98)$. Finally, due to differences between instruction group and no instruction group in percentage of time spent on mode frequency, a $T$-Test was performed comparing the mean of both groups on that dependent variable.

\section{Results}

\subsection{Effects of velocity, instruction and cue type}

As was expected, a significant interaction effect was obtained between treadmill velocity and step frequency $\left(F_{(3,21)}=402,191, p=.001, \varepsilon=.655,{ }^{1}\right.$ partial $\left.\eta^{2}=.983\right)$, indicating that step frequency increased linearly with treadmill velocity. However, this interaction was not affected by instruction (interaction velocity $\times$ instruction: $F_{(3,21)}=.764, p=.482, \varepsilon=.655$, partial $\left.\eta^{2}=.098\right)$. This is evident in Fig. 1 , which shows the effects of cue value on average step frequency (in $\mathrm{Hz}$ ) for all treadmill velocities, and for both experimental groups.

In the instruction group (Fig. 1A), the step frequency for the cue value of $100 \%$ overlapped with that of the preliminary tests for all participants at all treadmill velocities. For all cue values and across all

\footnotetext{
${ }^{1}$ Represents the Epsilon - Greenhouse-Geisser, with the correction factor for the degrees of freedom.
} 

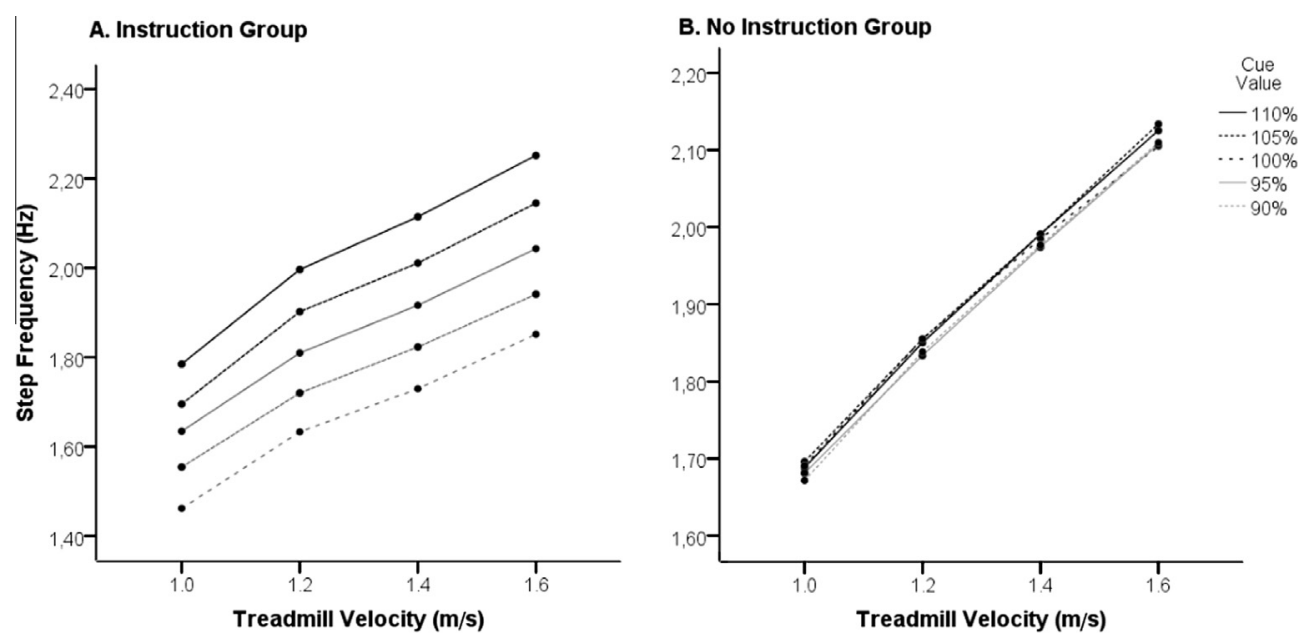

Fig. 1. Interaction between treadmill velocity and step frequency. Results plotted by auditory cue value (90\%, $95 \%, 100 \%, 105 \%$ and $110 \%$ of baseline frequency). (A) Plots results from the instruction group; (B) plots results from the no instruction group. The effect of the auditory cue is strongly affected by the instruction.

velocities, the distribution of step frequency was homogeneous and proportional to the cue value step frequency increased for cue values above $100 \%$ and decreased for values below. As for the no instruction group (Fig. 1B), although the step frequency increased linearly with treadmill velocity, it remained virtually unaffected by cue value. In fact, the average step frequency overlapped with the baseline values obtained in the preliminary test. The average standard deviation in the instruction group was .11, whereas in the no instruction group it was .09, revealing a small inter-subject variability.

On average the instruction group showed lower step frequencies than the no instruction group (cf. Fig. 2) across all treadmill velocities, but this difference proved not to be significant $\left(F_{(1,7)}=.105\right.$, $p=.755)$. The effect of cue type over step frequency was also not significant $\left(F_{(1,7)}=.869, p=.382\right.$, $\varepsilon=1.00$, partial $\eta^{2}=.110$ ) and was not affected by instruction (interaction cue type $\times$ instruction: $F_{(1,7)}=.048, p=.833, \varepsilon=1.00$, partial $\left.\eta^{2}=.007\right)$.

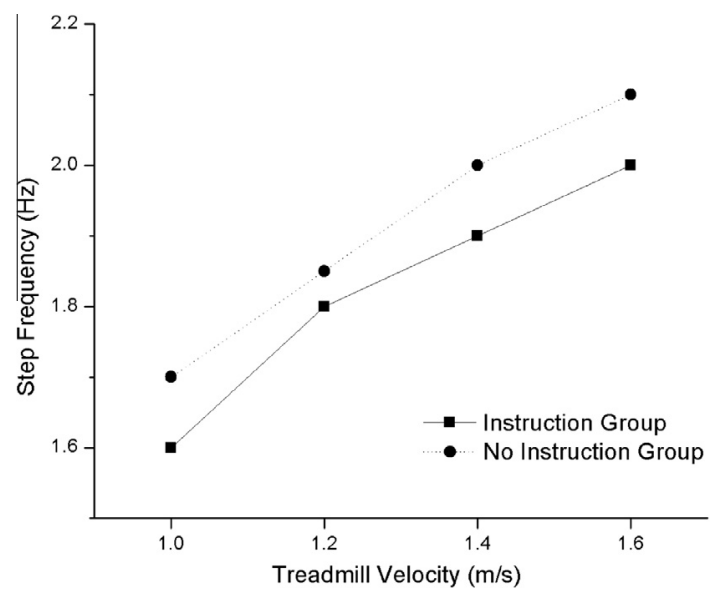

Fig. 2. Distribution of the mean step frequency. Mean step frequency in the instruction group and in the no instruction group as a function of treadmill velocity (all cue values averaged). The effect of velocity over step frequency is unaffected by instruction. 


\subsection{Effect of cue frequency value}

As was reported earlier, step frequency is affected by cue value in the instruction group, which indicates that participants were able to adapt their gait during the presentation of the stimuli. In this section, we intended to explore how this was accomplished by analyzing the correlation between cue value and average frequency.

Hence, we plotted cue value and step frequency in a normalized scale, having as reference what was named baseline frequency - the step frequency during the $15 \mathrm{~s}$ prior to the introduction of the auditory stimuli. Note that here the baseline frequency of $100 \%$ was not the one determined in the preliminary test, but the one based on initial step frequency of each trial.

Fig. 3 clearly shows that there was an effect of the cue value on step frequency for the instruction group, but not for the no instruction group. In the instruction group there was a strong correlation between cue value and step frequency for both step sounds $\left(R^{2}=.97, F=268, p \leqslant .001\right)$ and music sounds $\left(R^{2}=.99, F=724, p \leqslant .001\right)$. With step sounds, the amount of variance in step frequency explained by cue value is slightly higher (97\%) than with music stimuli (94\%), which reflects the higher standard deviation and frequency variability in the former. Still, both linear regressions were fitted with a slope of 1.0, which confirms that, in this group, step frequency increases proportionally with cue value. As for the no instruction group, the data for both stimuli yielded good fits with regression values close to zero (step: $R^{2}=.18, F=18.6, p \leqslant .001$; music: $R^{2}=.1, F=9.8, p \leqslant .005$ ). Hence, there was no effect of type of stimuli on step frequency. Moreover, there appears to be no specific tendency associated with cue value, with fitting slopes also close to zero for both types of stimuli (music: .06, step: .08).

In sum, these data reveal that participants in the instruction group matched their average step frequency with cue value for both music and step sounds, whereas in the no instruction group they did not.

\subsection{Step frequency variations over time}

This section analyzes step frequency patterns over time. Each $90 \mathrm{~s}$ trial was divided into 18 fivesecond blocks, and step frequencies were calculated for each interval. Subsequently, we extracted

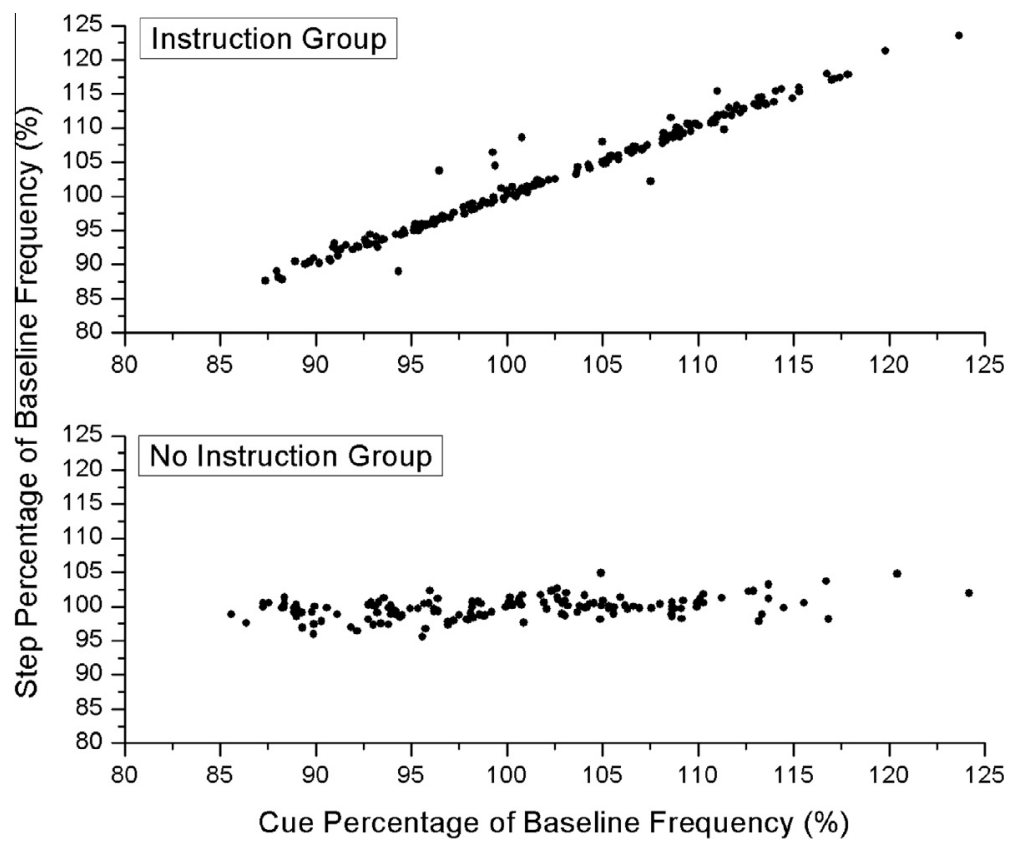

Fig. 3. Step frequency percentage as a function of cue percentage for both experimental groups and both sound types. 
the mode frequency for each trial and calculated for how long step frequencies remained constant. This was used as a measure of stride stability. These results are presented as percentage of time spent on mode frequency in Table 1.

On average, the instruction group remained in the mode step frequency for longer $(72.9 \%$ of the time) than the no instruction group (66.7\%), although these differences were not statistically significant in a $T$-Test $\left(t_{7}=1.27\right.$, n.s. $)$.

As for the type of stimuli, results showed that participants in the instruction group remained longer in mode step frequency with music stimuli (73.9\%) than with step sounds (71.9\%), whereas there were no such differences in the no instruction group.

Lastly, cue value affected stride stability in both groups: cue values of $90 \%, 95 \%$ and $110 \%$ led to less time spent in mode step frequency when compared to the remaining cue values for the instruction group. In the no instruction group, less time was spent in mode frequency for cue values of $105 \%$ and $110 \%$.

In order to better understand these analyzes, Fig. 4 exemplifies the step frequency distribution over time for two participants, one from each experimental group, with a cue value of $110 \%$ and walking at a treadmill velocity of $1.4 \mathrm{~m} / \mathrm{s}$.

In the instruction group, Fig. 4A demonstrates that this participant's step frequency distribution varied around the cue value, never deviating more than $.1 \mathrm{~Hz}$. Moreover, these variations appear to follow some type of pattern, since there were 9 periods of time in which step frequency was above cue value, and 9 periods below.

On the other hand, Fig. 4B clearly shows that the participant from the no instruction group followed a step frequency far from the cue value. As with the other group, small frequency variations were present.

These small variations were further explored in a frequency fluctuation analysis that revealed that while the instruction group fluctuates around the cue value presented, participants in the no instruction group vary around a value unrelated to the stimulus, close to their comfort value (as shown in Fig. 1B). Regardless of why, path stability is more constant and fluctuation decreases when there is a clear instruction to synchronize.

\section{Discussion}

The primary goal of this study was to assess if intention is required to observe an effect of external rhythmic cues while walking on treadmill, either by synchronization or by motor stabilization. As a secondary goal, this work also intended to compare rhythmic human steps and music on sound induced gait changes. Intention was manipulated through instruction, with one group being instructed to synchronize their gait with the auditory stimuli, and the other group merely being instructed to walk. Moreover, participants walked at different treadmill velocities, with the two types of cues combined in different frequency values.

Table 1

Percentage of time participants walked at mode frequency (0-100\%): average, standard deviation, minimum and maximum values obtained across participants.

\begin{tabular}{|c|c|c|c|c|c|c|c|c|c|c|}
\hline \multirow[t]{2}{*}{ Cue } & \multicolumn{5}{|c|}{ Step cue } & \multicolumn{5}{|c|}{ Music cue } \\
\hline & $90 \%$ & $95 \%$ & $100 \%$ & $105 \%$ & $110 \%$ & $90 \%$ & $95 \%$ & $100 \%$ & $105 \%$ & $110 \%$ \\
\hline \multicolumn{11}{|c|}{ Instruction group } \\
\hline Average & 69.2 & 70.8 & 72.2 & 75.8 & 71.7 & 70.6 & 70.6 & 76.4 & 80.8 & 70.8 \\
\hline SD & 14.9 & 12.1 & 14.8 & 14.2 & 16.7 & 15.3 & 13.8 & 13.2 & 15.1 & 16.4 \\
\hline Min & 50.0 & 50.0 & 50.0 & 50.0 & 44.4 & 50.0 & 44.4 & 55.6 & 50.0 & 33.3 \\
\hline Max & 94.4 & 88.9 & 100.0 & 100.0 & 100.0 & 100.0 & 94.4 & 100.0 & 100.0 & 94.4 \\
\hline \multicolumn{11}{|c|}{ No instruction group } \\
\hline Average & 67.4 & 68.8 & 62.5 & 62.8 & 71.9 & 68.3 & 69.1 & 61.8 & 66.3 & 68.8 \\
\hline SD & 18.0 & 11.1 & 11.0 & 18.0 & 16.8 & 18.0 & 18.7 & 13.4 & 17.3 & 11.8 \\
\hline Min & 38.9 & 50.0 & 44.4 & 33.3 & 38.9 & 38.9 & 38.9 & 44.4 & 44.4 & 50.0 \\
\hline Max & 100.0 & 88.9 & 83.3 & 100.0 & 100.0 & 100.0 & 100.0 & 88.9 & 100.0 & 94.4 \\
\hline
\end{tabular}



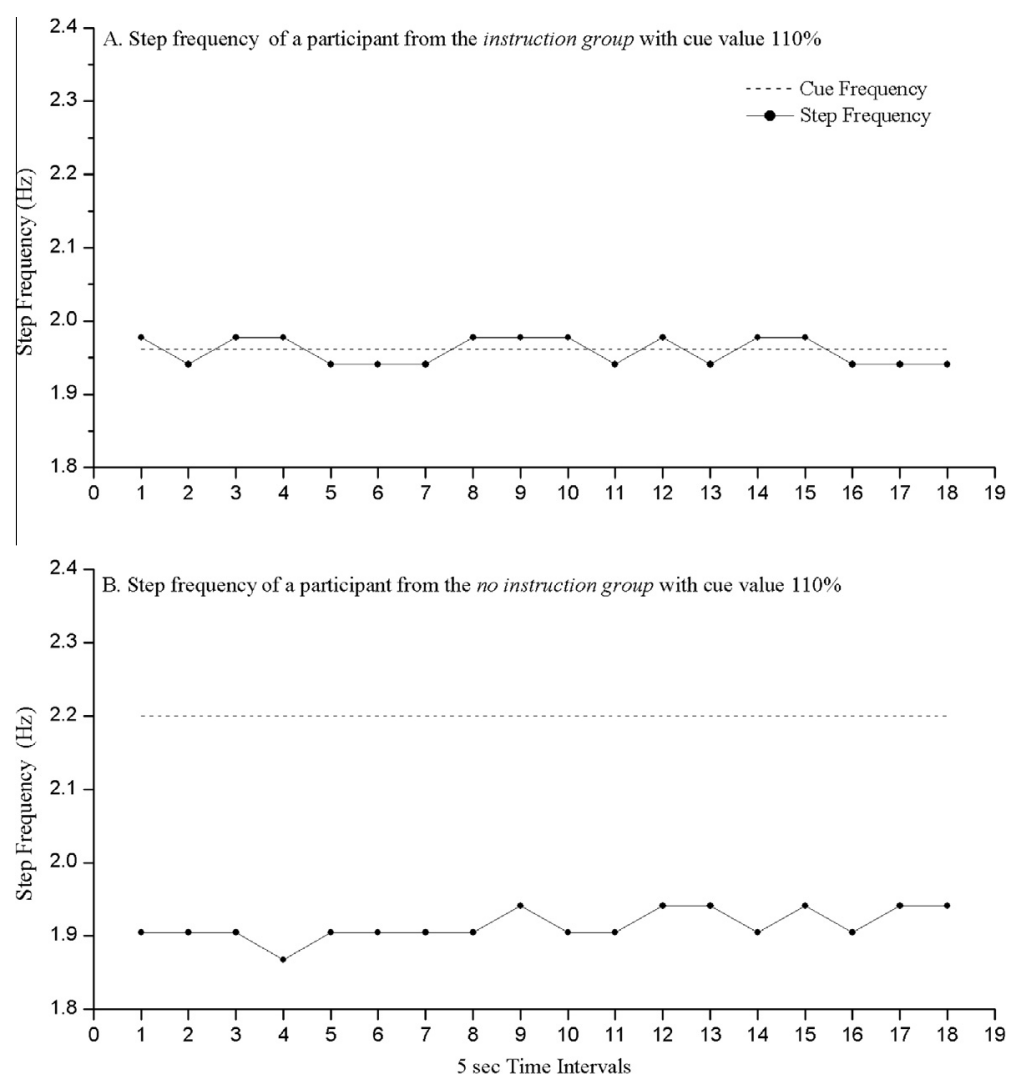

Fig. 4. Two examples of step frequency variations during a trial. Absolute values are plotted against frequency mode and stimulus frequency. (A) Presents data of a participant from the instruction group at cue value $110 \%$. (B) Presents data of a participant from the no instruction group at same cue value.

Both groups showed a linear increase in step frequency with velocity, thus confirming a significant effect of treadmill velocity over step frequency. Additionally, there was a general interaction between cue value and step frequency for the instruction group. This result is consistent with the hypothesis that the effect of auditory cues on the temporal characteristics of walking is independent of the treadmill's velocity (Bank, Roerdink, \& Peper, 2011;Osaki, Kumin \& Cihen, 2008; Picelli et al., 2010). Consequently, both treadmill velocity and sounds directly impact walking on a treadmill in an independent, dual way (Terrier \& Dériaz, 2011).

As to the primary goal of the study, it was found that the interaction between cue value and step frequency occurred only when participants were explicitly instructed to synchronize. This finding appears to differ from a previous study on treadmill walking that showed unintentional synchronization even with limited interpersonal stimulation (Nessler \& Gilliland, 2010). To analyze the effect of instruction and sound on motor stabilization, an analysis of gait frequency variation over time was also performed. Again, results revealed the importance of intention, since the instruction group synchronized in all trials for most of the time, whereas the no instruction group revealed barely any gait changes. Interestingly, the instruction group remained in the same step frequency for longer periods of time and showed lower step frequencies. However, both these differences were not statistically significant. In any case, results clearly reveal that, with explicit instructions to synchronize, a stable gait pattern is obtained for sustained periods, and this stable adaptation can stretch up to $+/-10 \%$ of the subject's natural walking frequency. 
In a secondary goal of this study, the effects of different sound types were analyzed. Researchers have studied the role played by music (Styns et al., 2007) and the metronome (Bank et al., 2011) in gait synchronization, but to the authors' knowledge, no study had ever compared the impact of walking-related step sounds and music. Surprisingly, in the present study no differences were found between trials with music or step sounds as stimuli. There was a higher variability in step frequency with music stimuli, but this effect was not statistically significant. It would have been expected that step sounds would activate social synchronization mechanisms, and consequently allow for spontaneous gait adaptation. However, our results were consistent across all participants, irrespective of instruction, which makes it all the more puzzling. Still, the fact that both sounds were associated with similar gait patterns in the instruction group reveals that both stimuli carry similar pacing information, regardless of their form or additional content.

In sum, the present study brings forth new insights on gait synchronization to auditory stimuli while walking on a treadmill. No differences were found between music and step sounds, which might signify that, for applied purposes, the type of sound used for stride stabilization could be irrelevant. The most surprising finding was the crucial effect of intention on gait synchronization. We suggest that, while walking on a treadmill, gait is only affected by auditory stimuli through attention and intention mechanisms. Hence, in applied settings, careful attention should be paid to the instructions given to the patients. Further research, preferably with larger samples, is needed to address the role of such cognitive mechanisms in gait adaptation.

\section{Acknowledgments}

This study was financed by the Portuguese Foundation for Science and Technology and FEDER (projects FCOMP-01-0124-FEDER-007560, FCOMP-01-0124-FEDER-022674, PEst-OE/ECI/UI4047/2011, PTDC/TRA/67859/2006 and PTDC/EEA-ELC/112137/2009), by the German Cluster of Excellence Hearing4all, and by the Academy of Finland project 13266239-Audiovisual Space.

The authors would like to thank Prof. Cristina Peixoto Santos, Ms. Corália Barbosa and Mr. Miguel Alexandre Campos Oliveira for the contributions on the data analysis algorithms.

\section{References}

Ada, L., Dean, C. M., Hall, J. M., Bampton, J., \& Crompton, S. (2003). A treadmill and overground walking program improves walking in persons residing in the community after stroke: A placebo-controlled, randimized trial. Archives of Physical Medicine and Rehabilitation, 84(10), 486-491.

Bank, P. J., Roerdink, M., \& Peper, C. E. (2011). Comparing the efficacy of metronome beeps and stepping stones to adjust gait: steps to follow! Experimental Brain Research, 209, 159-169.

de Bruin, N., Doan, J. B., Turnbull, G., Suchwersky, O., Bonfield, S., Hu, B., et al (2010). Walking with music is a safe and viable tool for gait training in Parkinson's disease: The effect of a 13-week feasibility study on single and dual task walking. Parkinson's Disease, 2010, 483530. http://dx.doi.org/10.4061/2010/483530.

Harris-Love, M. L., Forrester, L. W., Macko, R. F., Silver, K. H., \& Smith, G. V. (2011). Hemiparetic gait parameters in overground vs. treadmill walking. Neurorehabilitation and Neural Repair, 15(2), 105-112.

Nessler, J. A., \& Gilliland, S. J. (2009). Interpersonal synchronization during side by side treadmill walking is influenced by leg length differential and altered sensory feedback. Human Movement Science, 28, 772-785.

Nessler, J. A., \& Gilliland, S. J. (2010). Kinematic analysis of side-by-side stepping with intentional and unintentional synchronization. Gait \& Posture, 31, 527-529.

Osaki, Y., Kumin, M., \& Cohen, B. (2008). Relative contribution of walking velocity and stepping frequency to the neural control of locomotion. Experimental Brain Research, 185, 121-135.

Parvataneni, K., Ploegi, L., Olney, S. J., \& Brouwer, B. (2009). Kinematic, kinetic and metabolic parameters of treadmill versus overground walking in healthy older adults. Clinical Biomechanics, 24, 95-100.

Pelton, T. A., Johannsen, L., Chen, L., Chen, H., \& Wing, A. M. (2010). Hemiparetic stepping to the beat: Asymmetric response to metronome phase shift during treadmill gait. Neurorehabilitation E Neural Repair, 24(5), 428-434.

Picelli, A., Camin, M., Tinazzi, M., Vangelista, A., Cosentino, A., Fiaschi, A., et al (2010). Three-dimensional motion analysis of the effects of auditory cueing on gait pattern in patients with Parkinson's disease: a preliminary investigation. Neurological Sciences, 31, 423-430.

Rochester, L., Nieuwboer, A., Baker, K., Hetherington, V., Willems, A. M., Chavret, F., et al (2007). The attentional cost of external rhythmical cues and their impact on gait in Parkinson's disease: Effect of cue modality and task complexity. Journal of Neural Transmission, 114(10), 1243-1248.

Roerdink, M., Lamoth, C. J. C., Kwakkel, G., van Wieringen, P. C. W., \& Beek, P. J. (2007). Gait coordination after stroke: Benefits of acoustically paced treadmill walking. Physical Therapy, 87(8), 1009-1022.

Roerdink, M., Lamoth, C. J. C., van Kordelaar, J., Elich, P., Komijnenbelt, M., Kwakkel, G., et al (2009). Rhythm perturbations in acoustically paced treadmill walking after stroke. Neurorehabilitation E' Neural Repair, 23, 668-678. 
Sejdić, E., Jeffery, R., Kroonberg, A. V., \& Chan, T. (2012). An investigation of stride stationarity while listening to music or viewing television. Human Movement Science, 31(3), 695-706.

Styns, F., Van Noorden, L., Moelants, D., \& Leman, M. (2007). Walking on music. Human Movement Science, 26, $769-785$.

Terrier, P., \& Dériaz, O. (2011). Kinematic variability, fractal dynamics and local dynamic stability of treadmill walking. Journal of Neuroengineering and Rehabilitation, 8-12.

Thaut, M. H., Miltner, R., Lange, H. W., Hurt, C. P., \& Hoemberg, V. (1999). Velocity modulation and rhythmic synchronization of gait in Huntington's disease. Movement Disorders, 14(5), 808-819.

Zivotofsky, A. Z., Gruendlinger, L., \& Hausdorff, J. M. (2012). Modality-specific communication enabling gait synchronization during overground side-by-side walking. Human Movement Science, 31, 1268-1285.

Zivotofsky, A. Z., \& Hausdorff, J. M. (2007). The sensory feedback mechanisms enabling couples to walk synchronously: An initial investigation. Journal of Neuroengineering and Rehabilitation, 4, 28. http://dx.doi.org/10.1186/1743-0003-4-28. 